\title{
Pressure rupture and spontaneous perforation of the oesophagus
}

\author{
DOUGLAS H. CLARK AND H. I. TANKEL \\ From the Western Infirmary, Glasgow
}

EDITORIAL SYNOPSIS This paper describes cases of two contrasting types of spontaneous perforation of the oesophagus. One of them, described as a pressure rupture, was unusual insofar as the perforation was into the right pleura and high in the oesophagus.

In 1946, when Barrett reviewed all the recorded cases of spontaneous perforation of the oesophagus, he was able to collect 60 among which there were no survivors. He concluded his paper with the following remarks: 'In the byeways of surgery there can be few conditions more dramatic in their presentation and more terrible in their symptoms than spontaneous perforation of the oesophagus. No case has yet been treated successfully and diagnosis has only been achieved in a very few before death and yet there is no fundamental reason why this unsatisfactory position should not be improved in the future. Several things are essential to success; firstly a knowledge that the accident can and does occur; secondly a knowledge of the symptomatology; thirdly an early diagnosis. Given these, I am convinced that surgeons will be able to save some patients by combining the principles already well established in the cases of abdominal perforation, with those relevant to thoracotomy.' In the following year he confirmed the wisdom of his remarks by successfully treating a case by thoracotomy and suture of the perforation.

The condition has been known since it was first reported in 1724 by Boerhaave. In 1952, at a meeting of the American Association for Thoracic Surgery the total number of recorded cases was 134 with 27 survivals and by 1954 Moynihan was able to trace more than 200 published cases adding three further fatal cases. Paton (1957) reported five further fatal cases from the Royal Infirmary of Edinburgh.

Though the condition would appear to be relatively rare, it is probably more common than the literature indicates. A perusal of the pathological archives of any large hospital will probably reveal several cases of spontaneous perforation quite apart from perforation associated with oesophageal pathology such as carcinoma, oesophagitis, and ulcer- ation. During the 10-year period 1950-1959 there were six cases of spontaneous perforation in the Western Infirmary, Glasgow, undiagnosed during life.

The purpose of the present paper is to report two interesting examples of this condition in patients who survived, one diagnosed and treated by thoracotomy and suture, the other misdiagnosed but with spontaneous recovery. A further purpose is to reinforce once more Barrett's plea for early diagnosis and surgical treatment, and to remind both physicians and surgeons, especially in their inexperienced years, of its existence, of its lethal nature, and of the need for prompt surgical treatment.

\section{CASE REPORTS}

CASE 1 A male commercial traveller, aged 47 , ate a normal three-course lunch consisting of soup, steak pie and vegetables, sweet and coffee at 12 noon. Two hours later, while travelling on a Clyde car ferry he felt sick and vomited at 2 p.m. During the act of vomiting he was suddenly seized by severe upper abdominal pain which radiated to his back between his shoulder blades. The backache was as severe as the pain in the abdomen. He was seen by a doctor at his destination, 50 miles from Glasgow, who diagnosed a perforated duodenal ulcer and sent him back by ambulance to Glasgow. He was admitted to the Western Infirmary at 8 p.m.

On admission the patient complained of severe pain in the abdomen, especially in the epigastrium and also in the back. He had no shoulder tip pain. Breathing was restricted and there was a tinge of cyanosis in the lobes of the ears and the lips. The abdomen was rigid in the epigastrium and guarded in the lower quadrants. Clinical examination of the chest revealed a diminished R.M. at the right base. The blood pressure was $130 / 85 \mathrm{~mm}$. $\mathrm{Hg}$. Spontaneous perforation of the oesophagus was suspected and radiographs of the chest and abdomen were taken in the erect position, the former showing a 


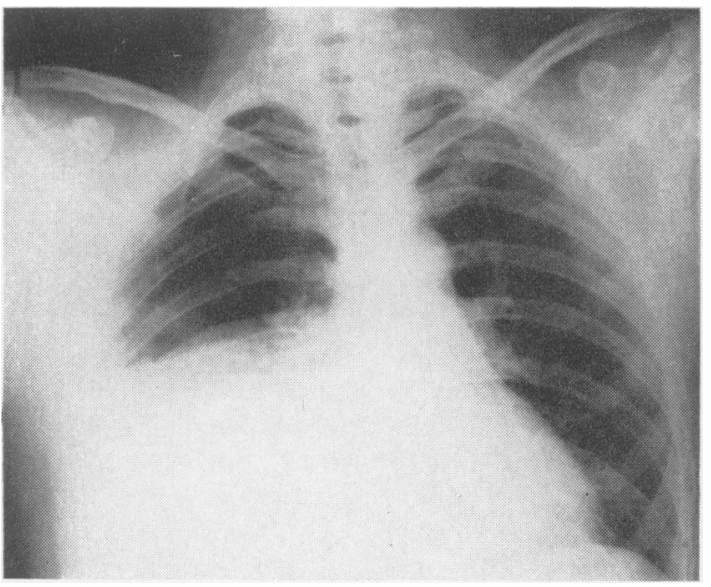

FIG. 1. Case 1. Radiograph of the chest showing hydropneumothorax on the right side.

moderate degree of pneumothorax and a small amount of fluid in the right side. There was no gas under the diaphragm (Fig. 1).

Seven hours after the perforation, the right chest was opened through the bed of the seventh rib. By this time, probably as a result of relaxation under anaesthesia, the pleural cavity contained about a litre of dirty, greyishgreen fluid with much of the lunch that he had eaten nine hours previously. There was a 2 in. longitudinal tear of the oesophagus immediately below the azygos vein. The pleural cavity was evacuated and the rent in the oesophagus closed with two layers of silk. The area was sealed thereafter by suturing lung to the oesophagus. The chest was closed with water-seal drainage. A gastrostomy was performed and constant suction maintained for 48 hours. Fluids and electrolytes were administered intravenously for three days, no fluid being allowed by mouth for two days. Antibiotic therapy consisted of tetracycline $250 \mathrm{mg}$. six-hourly given parenterally for five days. The chest drain was removed on the third day, the chest radiograph showing complete re-expansion of the lung. The gastrostomy tube was removed on the fifth day. Recovery thereafter was uneventful and swallowing was normal. Two months later a barium swallow and meal showed neither structural nor functional abnormality of the oesophagus, stomach, or duodenum.

The diagnostic point in this case was that the rupture of oesophagus took place during the act of vomiting. It is interesting that the patient later stated that he had always been afraid to vomit and furthermore on the rare occasions when he had vomited he felt as if 'something was going to give'. He felt that while vomiting 'he built up a large pressure because the exit could not cope'. Nevertheless, subsequent investigation showed the oesophagus to be normal in every way. A lack of coordination with failure of relaxation at the pharyngo-oesophageal junction cannot, however, be excluded.

The site of perforation, in this case immediately below the azygos vein, is unusual. Apart from one case reported by Moynihan (1954) all other recorded cases have been at the lower end of the oesophagus within a short distance of the hiatus, the perforation invariably contaminating the left pleural cavity.

Although this case resembled a perforated duodenal ulcer, the history of the onset of pain during vomiting enabled one of the authors to make the diagnosis before seeing the patient. This observation is made to emphasize the importance of the history and the distribution of the pain-the sudden onset of severe abdominal pain and backache during the act of vomiting. Several previous authors have made the diagnosis by telephone.

CASE 2 A postman aged 39, while asleep in bed, was suddenly seized in the early hours of the morning by severe pain all over the abdomen and through to the back. Thereafter rest was impossible and he 'walked the floor' until admitted to hospital five hours later. Though he was not normally subject to stomach trouble, indigestion, or heartburn, for four nights preceding this catastrophe his sleep had been disturbed by deep epigastric discomfort unrelieved by food or antacids.

On admission the abdomen was rigid in all areas and silent. The temperature was $102^{\circ} \mathrm{F}$ and the pulse rate 120 .

A perforated peptic ulcer was diagnosed, a laparotomy performed, but no intraperitoneal abnormality was found. The abdomen was closed.

Following operation he continued to have pain, fever, and tachycardia. By the following day a pleural rub was heard at the left base with crepitations on both sides. A chest radiograph showed partial collapse of the left lower lobe and appearances of pneumonia at the right base. Crystamycin was given twice daily with a reduction in both temperature and pulse rate.

He continued to complain of epigastric pain and backache. Three days after operation he began to complain of pain on swallowing and this continued with some severity for about 10 days, gradually improving. A barium swallow 12 days after operation revealed some extravasation of the barium from the oesophagus about $8 \mathrm{~cm}$. above the diaphragm. Neither hiatus hernia nor reflux could be demonstrated and the stomach and duodenum were normal. By this time there was a small pleural effusion in the left chest with a collapse of the left lower lobe affecting mainly the posterior basal segment (Fig. 2). During this time his general condition gave no cause for concern. Chloramphenicol was substituted for Crystamycin and the dysphagia gradually became less.

Eighteen days after admission oesophagoscopy was performed and at $32 \mathrm{~cm}$. there was thickening of the 


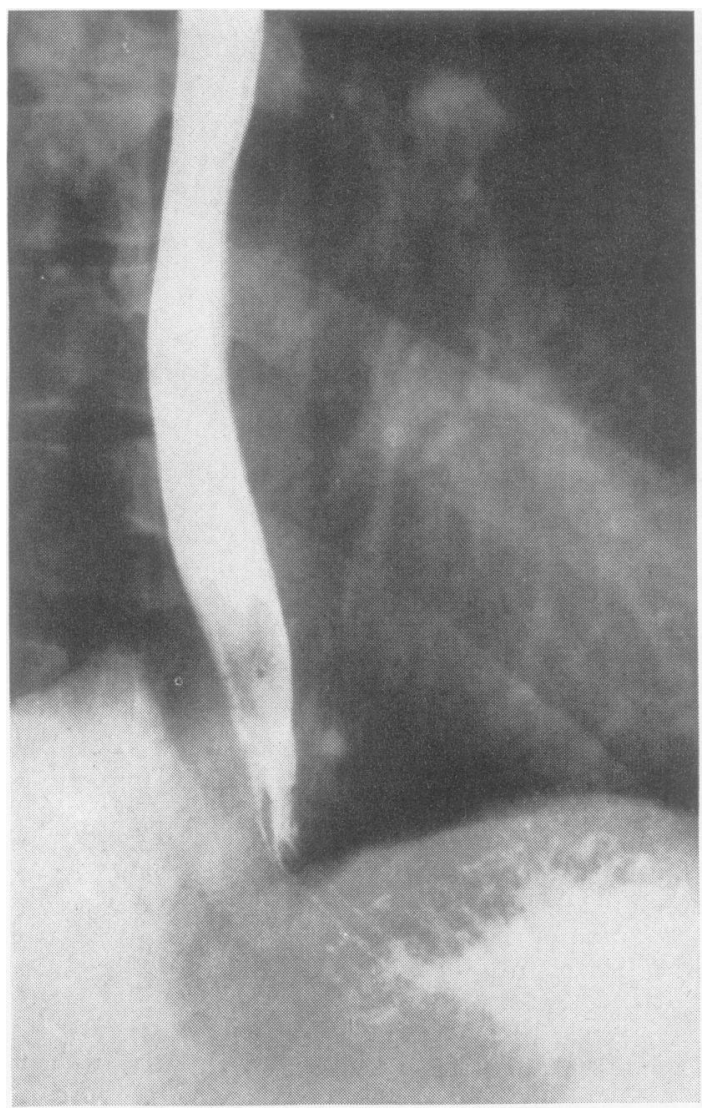

FIG. 2. Case 2. Barium swallow examination showing escape of a small amount of barium into the mediastinum to the left of the lower end of the oesophagus. The left pleura has remained intact.

mucosa and a little localized bleeding at a point thought to be the site of the perforation. The remainder of the oesophagus at that level and elsewhere appeared normal.

His further progress was uneventful though dysphagia continued in a mild form for about three months. Thereafter it disappeared and he is now symptom free.

This case differs from the previous one and from other typical cases of 'spontaneous' perforation in that the rupture occurred during sleep and without vomiting. Several cases have been reported where the rupture has occurred with such minor exertions as yawning, extending the arms above the head, and coughing. It is unlikely that such movements could precipitate rupture of the oesophagus. We believe that these cases, like the present case 2, are probably due to perforation of an acute penetrating ulcer of the lower oesophagus. In the present case the complaint of deep-seated epigastric pain for four nights before the perforation indicates this probability.

Recovery without active treatment was due no doubt in part to the effectiveness of the antibiotic therapy but mainly to the limited nature of the extravasation, the pleura remaining intact.

\section{DISCUSSION}

Spontaneous rupture of the oesophagus has been the subject of several excellent and comprehensive reviews both in Great Britain and in America (Barrett; 1946, Carter, Alrich, and Drash, 1951, Moynihan; 1954 and Paton; 1957). Some authors have expressed their dissatisfaction with the term 'spontaneous rupture of the oesophagus' and confusion has arisen by grouping together cases of different aetiology. Excluding perforation associated with carcinoma of the oesophagus, it is now becoming evident that there are two distinct types of perforation which are exemplified by the two cases described. The type of rupture of the oesophagus, of which case 1 is a typical example, is the result of an overwhelming build-up of pressure within the oesophagus during the act of vomiting and the word 'spontaneous' can hardly apply. Moynihan (1954) has suggested the term 'pressure rupture of the oesophagus' which indicates exactly what has taken place, and for this reason it ought to be adopted. Its descriptive nature has the added advantage of conveying the explosive nature of the lesion, the possible widespread contamination, and the urgency of surgical treatment. Indeed it would appear from collective experience that it is in these cases that gross involvement of one or other pleural cavity occurs.

Case 2 is probably an example of true 'spontaneous' perforation of the oesophagus in the same sense that a perforation of a duodenal ulcer is 'spontaneous'. Violent pressure build-up is not essential though no doubt in some cases it has been the final perforating factor. Sandry (1962), in a histological study of surgically excised specimens of oesophagitis, has shown that acute and subacute penetrating ulcers of the lower oesophagus are not uncommon alone or in association with superficial oesophagitis. He has also shown that these ulcers are situated in islands or encroachments of mucosa of gastric cardiac type and therefore are closely akin to the ulcers described by Barrett (1950). Lacking the high pressure force of vomiting, perforation in these cases may lead only to limited contamination of the mediastinum and the pleura may remain intact. This second case and others reported have occurred without convincing evidence of increased intra-oesophageal pressure. Though recovery ensued 
on conservative treatment, we are in no doubt that early surgical treatment offers more certain and rapid cure.

The relationship of the Mallory-Weiss syndrome to pressure rupture of the oesophagus may be one of degree. Both conditions are frequently associated with the vomiting of a large volume of gastric contents associated most often with excessive eating and drinking. In the former condition the force is generally insufficient to rupture all the coats of the oesophagus though two of the 11 patients described by Atkinson, Bottrill, Edwards, Mitchell, Peet, and Williams (1961) died from perforation of the oesophagus. Nevertheless in Mallory-Weiss cases the mucosal fissures are mainly on the gastric side of the oesophago-gastric junction and bleeding is the prominent complication. The effect of pressure may be localized to the fundus of the stomach because of hiatus hernia or mucosal atrophy, both of which conditions were found in more than half of the cases described by Atkinson and his colleagues.

The clinical features of perforation of the oesophagus have been dramatically described in previous publications and there is little need to dwell on them again. However, since the main purpose of this paper is to promote clinical awareness of the condition and its grave consequences we should like once more to stress the salient features. Though the condition tends to occur in the youngish or middle-aged man, alcoholic and dietetic indiscretion has a wide range, and a considerable proportion of the reported cases have been in the older age groups. Indeed as in the 'pressure rupture' reported here, overindulgence is not essential. The sudden onset of severe abdominal or retrosternal pain associated with severe backache coming on during or after the act of vomiting should arouse a clinical suspicion that is not dispelled until appropriate investigation rules out the possibility of oesophageal perforation. The onset of similar pain without vomiting requires similar vigilance and adequate investigation. The danger lies in the patient being labelled, on the one hand, by physicians as a case of myocardial infarct, spontaneous pneumothorax, or dissecting aneurysm, and by surgeons on the other hand as of perforated peptic ulcer or pancreatitis. We suggest that such patients should have radiographs of the abdomen and chest in the erect position to demonstrate gas under the diaphragm, mediastinal emphysema, or the presence of air or fluid in the chest. If the diagnosis is still in doubt the $x$-ray examination should be completed by the administration of a small amount of gastrografin to demonstrate extravasation.

When laparotomy has been mistakenly undertaken we are of the opinion that the patient's chest should be radiographed on the operating table so that thoracotomy may then be performed if necessary.

\section{SUMMARY}

Two cases of perforation of the oesophagus are described, one a pressure rupture and the other a spontaneous perforation of an acute penetrating ulcer.

Perforation of the oesophagus should always be considered in cases presenting with the sudden onset of severe abdominal pain and backache. When this onset is associated with vomiting, perforation of the oesophagus is highly probable.

The importance of early diagnosis and surgical treatment is stressed and in most cases offers the only hope of recovery.

The term 'pressure rupture' should be adopted for those cases in which perforation occurs during the act of vomiting.

We wish to thank Mr. Kenneth Fraser for his constructive criticisms in the preparation of this paper and the X-ray and Medical Illustration Departments for the illustrations.

\section{REFERENCES}

Atkinson, M., Bottrill, M. B., Edwards, A. T., Mitchell, W. M., Peet, B. G., and Williams, R. E. (1961). Mucosal tears at the oesophagogastric junction (the Mallory-Weiss syndrome). Gut, 2, 1-11.

Barrett, N. R. (1946). Spontaneous perforation of the oesophagus. Thorax, 1, 48-70.

(1947). Report of a case of spontaneous perforation of the oesophagus successfully treated by operation. Brit. J. Surg., 35, 216-218.

- (1950). Chronic peptic ulcer of the oesophagus and 'oesophagitis' Ibid., 38, 175-182.

Boerhaave, H. (1724). Atrocis, nec descripti prius, morbi historia secundum medicae artis leges conscripta. Boutestein Leyden. Quoted by Moynihan, N. H. (1954). Lancet, 2, 728-732.

Carter, J. P., Alrich, E. M., and Drash, E. C. (1951). Spontaneous rupture of the esophagus. Surgery, 30, 500-505.

Moynihan, N. H. (1954). Pressure perforation and rupture of the oesophagus. Lancet, 2, 728-732.

Paton, B. C. (1957). Spontaneous rupture of the oesophagus. Scot. med. J., 2, 97-104.

Sandry, R. J. (1962). The pathology of chronic oesophagitis. Gut, 3, 189-200. 